\title{
Prognostic role of phospho-PRAS40 (Thr246) expression in gastric cancer
}

Yi-Zhuo Lu' ${ }^{1}$ An-Mei Deng ${ }^{2}$, Liang-Hui Li ${ }^{1}$, Guo-Yan Liu' ${ }^{1}$, Guo-Yang Wu

1Department of General Surgery, Zhongshan Hospital, Research Institute of Digestive Diseases, Xiamen University, Xiamen, Fujian Province, China

2Department of Laboratory Diagnosis, Changhai Hospital, Second Military Medical University, Shanghai, China

Submitted: 19 October 2011

Accepted: 14 December 2011

Arch Med Sci 2014; 10, 1: 149-153

DOI: 10.5114/aoms.2013.36927

Copyright @ 2014 Termedia \& Banach
Corresponding author:

Guo-Yang Wu

Department of General

Surgery, Zhongshan Hospital,

Research Institute of

Digestive Diseases Xiamen

University, Xiamen Fujian

Province, China

Phone: 0592-2993161

E-mail:

13515965398@163.com

\begin{abstract}
Introductions: Phospho-PRAS40Thr246 (phosphorylated proline-rich Akt substrate of 40 kilodaltons at Thr246) is a biomarker for phosphatidylinositol 3-kinase (PI3K) pathway activation and AKT inhibitors sensitivity.

Material and methods: In this study, we immunohistochemically investigated the expression of phospho-PRAS40Thr246 in 141 gastric cancer tumors, and evaluated its clinicopathological and prognostic significance.

Results: Sixty-four cases (45.4\%) were defined as phospho-PRAS40Thr246 positive. Phospho-PRAS40Thr246 correlated positively with lymph node metastasis, lymphatic infiltration, vascular infiltration and shorter survival. Furthermore, phospho-PRAS40Thr246 is an independent prognostic factor for gastric cancer. Conclusions: Our data suggest that phospho-PRAS40Thr246 was frequently expressed in gastric cancers, and correlated with malignant progression and poor prognosis of patients. PI3K pathway-targeted therapies should be considered in the future treatment of gastric cancers.
\end{abstract}

Key words: PRAS40, phosphorylation, PI3K, prognosis, targeted therapy.

\section{Introduction}

Gastric carcinoma remains one of the most common digestive malignancies in the world, especially in Asia, including China [1]. Radical surgical resection with adequate lymph node dissection is the only curative treatment for localized gastric cancer; currently available adjuvant chemotherapies have limited effects on survival. Therefore, novel specific agents that successfully target specific "oncopathways" in cancers are also being considered in the treatment of gastric cancer.

The phosphatidylinositol 3-kinase (PI3K) pathway is frequently activated in human tumors including gastric cancer, which is usually caused by mutations in tumor suppressor gene PTEN or p110-a catalytic subunit of PI3K, or triggered by receptor tyrosine kinases (RTKs) signaling [2-4]. It has been speculated that activation of the PI3K pathway plays a crucial role in the process of cancer development and maintenance [5]. Therefore, this pathway might be an attractive target for cancer therapy. Accordingly, small-molecule inhibitors targeting PI3K and other components of PI3KAKT-mTOR pathways are currently under active clinical development [6]. However, currently used biomarkers for measuring PI3K pathway activation and predicting the efficacy of its inhibitors are inadequate, which lim- 
its the utility of PI3K pathway inhibitors in the personalized treatment of cancer [7].

Most recently, Andersen et al. measured the phosphoprotein modification in specific proteins accompanying PI3K pathway activation, and identified phospho-PRAS40 Thr246 is a useful biomarker to measure PI3K pathway activation and predict AKT inhibitor sensitivity in multiple cancers [8]. Furthermore, compared with other epitopes, phosphoPRAS40 Thr246 is highly stable in tissue samples and thus is ideal for detection by immunohistochemistry [8].

Several previous studies have reported the expression of phospho-PRAS40 Thr246 in various human cancers [9-11]. However, the expression of phospho-PRAS40 Thr246 in gastric cancer, especially as a novel biomarker for PI3K pathway activation and inhibitor response, has not been investigated.

Thus, the purpose of this study was to examine phospho-PRAS40 Thr246 in gastric cancer and evaluate its clinicopathological and prognostic significance, and to further evaluate which subgroup of patients with gastric cancer would benefit from therapies targeting the PI3K pathway.

\section{Material and methods}

\section{Patients and specimens}

One hundred and forty-one patients with histologically confirmed gastric adenocarcinoma were retrospectively collected in this study. All patients underwent radical gastrectomy with lymph node dissection at Zhongshan Hospital, Xiamen University, China and Changhai Hospital, Second Military Medical University, China between January 2006 and May 2008. Patients undergoing neo-adjuvant chemotherapy and/or radiotherapy were not included. All the patients received postoperative adjuvant chemotherapy based on leucovorin and 5-FU. The pathological tumor staging was determined by the TNM classification, and historical grading of each cancer tissue was performed according to the WHO criteria. Tumor histologies were classified into the differentiated type (papillary, well or moderately differentiated adenocarcinoma, well-differentiated mucinous carcinoma) and the undifferentiated type (poorly or undifferentiated adenocarcinoma, signet

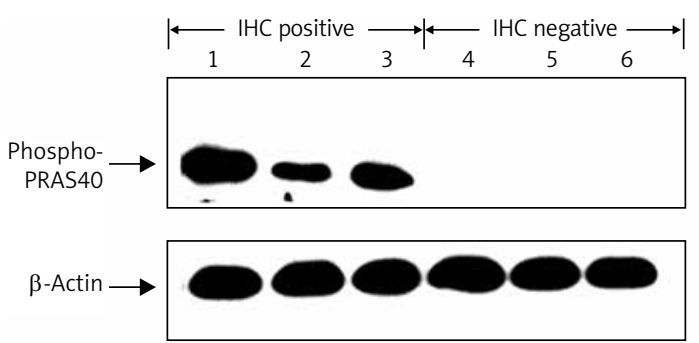

Figure 1. Results of immunohistochemical analysis corresponding to those of Western blot analysis ring cell carcinoma and poorly differentiated mucinous carcinoma). The study was approved by our local ethics committees.

\section{Immunohistochemistry (IHC)}

Immunohistochemistry was performed using the streptavidin-biotin complex method. Rabbit mAb against phospho-PRAS40 (Thr246) (Cell Signaling, C77D7) was used as a primary antibody at a working dilution of $1: 100$. Briefly, $4 \mu \mathrm{m}$ thick formalinfixed paraffin-embedded tissue sections were deparaffinized in xylene and hydrated in graded alcohols. After antigen retrieval in $0.01 \mathrm{M}$ citrate buffer ( $\mathrm{pH}$ 6.0), the endogenous peroxidase activity of specimens was blocked by $0.3 \% \mathrm{H}_{2} \mathrm{O}_{2}$ for $10 \mathrm{~min}$ at room temperature. Sections were incubated with the primary antibody at $4^{\circ} \mathrm{C}$ overnight, then were washed three times and treated with secondary antibody at room temperature for $2 \mathrm{~h}$. After washing, the specimens were treated with $A B C$ reagent (Dako), followed by color development with DAB and counterstaining with haematoxylin. Negative controls were carried out by substituting normal serum for the primary antibody, and the positive controls were human breast adenocarcinoma tissues. Immunostaining results were evaluated based upon the percentage of visible cancer cells that stained positive. According to the criteria established in the previous studies, cases with the percentage of positively staining cells greater than $10 \%$ were defined as positive.

\section{Western blot}

Frozen tissues of 6 primary gastric carcinoma knowing $\mathrm{IHC}$ results (3 $\mathrm{IHC}$ positive and 3 negative) were homogenised in lysis buffer. Immunodetection was done with the same antibody used in immunohistochemistry analysis (at 1: 1000 dilutions) with an enhanced chemiluminescence system (Amersham). The membrane was stripped and reblotted with $\beta$-actin antibody (Santa Cruz Biotech) at $1: 1000$ dilutions as a control.

\section{Statistical analysis}

The association between phospho-PRAS40 Thr 246 and clinicopathological parameters was assessed using Student's $t$ test or $\chi^{2}$ test where appropriate. The overall survival (OS) time was defined as the interval between resection and death or date last known alive. Kaplan-Meier methods were used to estimate overall survival, and differences were determined by the log-rank test. Multivariate analysis was carried out using the Cox regression method. Values of $p<0.05$ were considered to be statistically significant. All statistical analyses were performed using SPSS 13.0 software. 

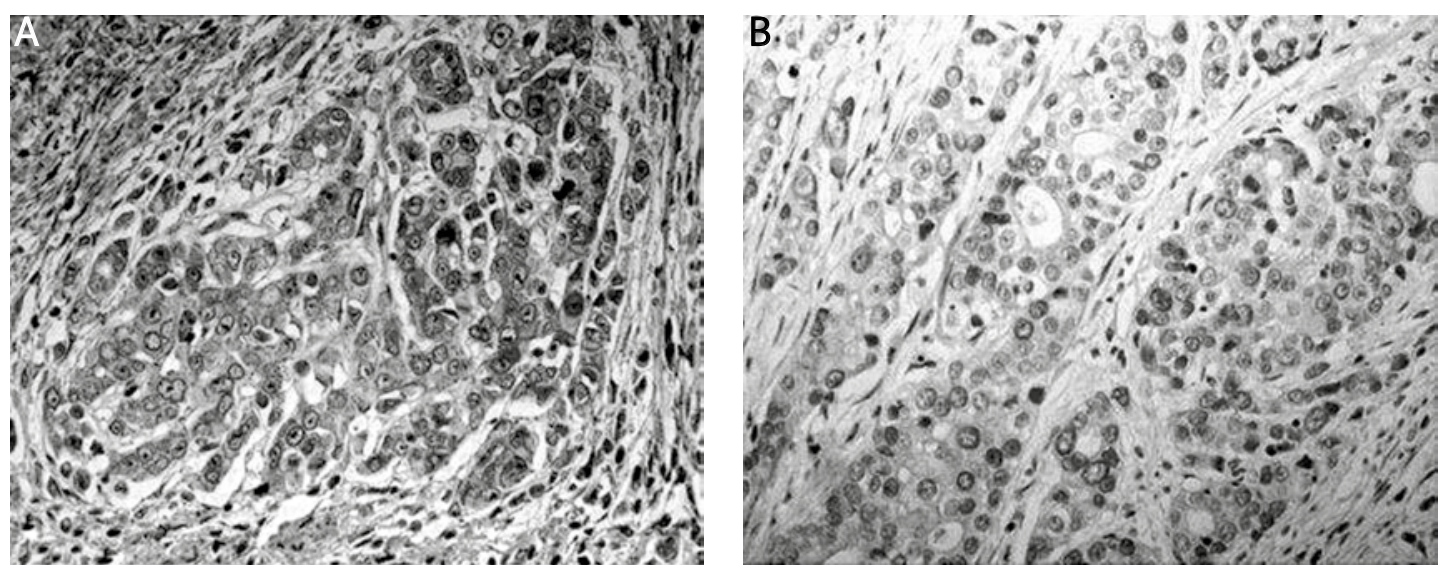

Figure 2. Immunoreactivity for phospho-PRAS40Thr246 in gastric cancer tissues: A - positive expression, B - negative expression

\section{Results}

We detected the expression of phosphoPRAS40 ${ }^{\text {Thr246 }}$ in primary gastric cancer tissues by immunohistochemistry and Western blot. Figure 1 shows the results of Western blot analysis, corresponding to those of immunohistochemistry. In gastric cancer specimens, phospho-PRAS40Thr246 staining was observed in the cytoplasm of cancer cells; occasionally membranous staining was also observed. No positive staining was identified in the surrounding stroma (Figure 2). According to the criteria specified, 64 of 141 tumors (45.4\%) were defined as phospho-PRAS40 Thr246-positive.

The correlation between the clinicopathological characteristics of patients with gastric cancer and the status of phospho-PRAS40 Thr246 expression is summarized in Table I. Lymph node metastases were significantly greater in the phosphoPRAS40 Thr246-positive group than in the negative group (79.7\% vs. 59.7\%, $p=0.011)$. Moreover, phos-

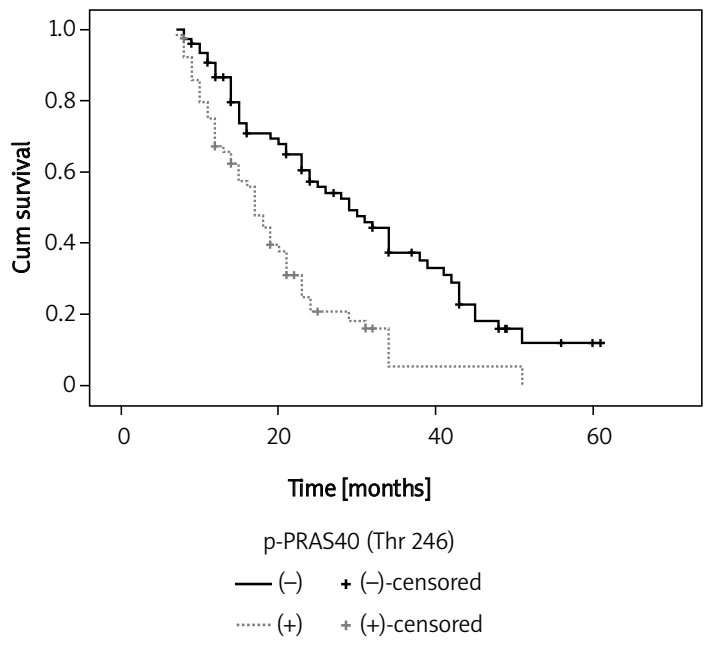

Figure 3. Overall survival curves in different groups of phospho-PRAS40Thr246 expression ( $p=0.001$, by log-rank analysis)
pho-PRAS40 Thr246 expression was also significantly higher in the tumors with positive lymphatic or vascular infiltration than those without lymphatic

Table I. Correlation between expression of phosphoPRAS40Thr246 and clinical factors

\begin{tabular}{|c|c|c|c|}
\hline \multirow[t]{2}{*}{ Clinical factors } & \multicolumn{2}{|c|}{$\begin{array}{c}\text { Expression of } \\
\text { phospho-PRAS40 Thr246 }\end{array}$} & \multirow[t]{2}{*}{ Value of $p$} \\
\hline & $\begin{array}{l}\text { Positive } \\
(n=64)\end{array}$ & $\begin{array}{l}\text { Negative } \\
(n=77)\end{array}$ & \\
\hline \multicolumn{4}{|l|}{ Age [year] } \\
\hline$<65$ & 47 & 54 & 0.664 \\
\hline$\geq 65$ & 17 & 23 & \\
\hline \multicolumn{4}{|l|}{ Gender } \\
\hline Male & 42 & 53 & 0.686 \\
\hline Female & 22 & 24 & \\
\hline \multicolumn{4}{|l|}{ Depth } \\
\hline $\mathrm{T} 1 / \mathrm{T} 2$ & 9 & 18 & 0.162 \\
\hline $\mathrm{T} 3 / \mathrm{T} 4$ & 55 & 59 & \\
\hline \multicolumn{4}{|c|}{ Lymph node metastasis } \\
\hline Yes & 51 & 46 & 0.011 \\
\hline No & 13 & 31 & \\
\hline \multicolumn{4}{|l|}{ Histology } \\
\hline Differentiated & 33 & 45 & 0.413 \\
\hline Undifferentiated & 31 & 32 & \\
\hline \multicolumn{4}{|l|}{ TNM stage } \\
\hline $1 / 2$ & 24 & 26 & 0.645 \\
\hline $3 / 4$ & 40 & 51 & \\
\hline \multicolumn{4}{|c|}{ Lymphatic infiltration } \\
\hline Yes & 50 & 42 & 0.003 \\
\hline No & 14 & 35 & \\
\hline \multicolumn{4}{|c|}{ Vascular vessel infiltration } \\
\hline Yes & 44 & 39 & 0.028 \\
\hline No & 20 & 38 & \\
\hline
\end{tabular}


Table II. Univariate and multivariate analysis with regard to overall survival

\begin{tabular}{|c|c|c|c|}
\hline \multirow[t]{2}{*}{ Parameter } & \multirow{2}{*}{$\begin{array}{c}\begin{array}{c}\text { Univariate } \\
\text { analysis }\end{array} \\
\text { Value of } p\end{array}$} & \multicolumn{2}{|c|}{$\begin{array}{l}\text { Multivariate } \\
\text { analysis }\end{array}$} \\
\hline & & Value of $p$ & RR $(95 \% \mathrm{Cl})$ \\
\hline \multicolumn{4}{|l|}{ Age [years] } \\
\hline$<65$ vs. $\geq 65$ & 0.650 & 0.457 & $\begin{array}{c}1.188 \\
(0.755-1.868)\end{array}$ \\
\hline \multicolumn{4}{|l|}{ Gender } \\
\hline Male vs. female & 0.531 & 0.642 & $\begin{array}{c}1.115 \\
(0.706-1.760)\end{array}$ \\
\hline \multicolumn{4}{|l|}{ Depth } \\
\hline T1/T2 vs. T3/T4 & 0.123 & 0.065 & $\begin{array}{c}0.540 \\
(0.281-1.039)\end{array}$ \\
\hline \multicolumn{4}{|c|}{ Lymph node metastasis } \\
\hline Yes vs. no & $<0.001$ & $<0.001$ & $\begin{array}{c}3.597 \\
(1.875-6.902)\end{array}$ \\
\hline \multicolumn{4}{|l|}{ Histology } \\
\hline $\begin{array}{l}\text { Differentiated vs. } \\
\text { undifferentiated }\end{array}$ & 5. 0.303 & 0.579 & $\begin{array}{c}1.119 \\
(0.753-1.664)\end{array}$ \\
\hline \multicolumn{4}{|l|}{ TNM stage } \\
\hline $1 / 2$ vs. $3 / 4$ & 0.006 & 0.187 & $\begin{array}{c}1.375 \\
(0.857-2.205)\end{array}$ \\
\hline \multicolumn{4}{|l|}{ Vascular infiltration } \\
\hline Yes vs. no & 0.035 & 0.109 & $\begin{array}{c}1.495 \\
(0.914-2.447)\end{array}$ \\
\hline \multicolumn{4}{|c|}{ Lymphatic vessel infiltration } \\
\hline Yes vs. no & 0.041 & 0.995 & $\begin{array}{c}1.002 \\
(0.616-1.628)\end{array}$ \\
\hline \multicolumn{4}{|c|}{ Phospho-PRAS40Thr246 } \\
\hline $\begin{array}{l}\text { Positive vs. } \\
\text { negative }\end{array}$ & $<0.001$ & 0.004 & $\begin{array}{c}1.883 \\
(1.231-2.880)\end{array}$ \\
\hline
\end{tabular}

or vascular infiltration $(78.1 \%$ vs. $54.5 \%, p=0.003$ $68.8 \%$ vs. $50.6 \%, p=0.028$, respectively). However, phospho-PRAS40 ${ }^{\text {Thr246 }}$ expression did not correlate with age, gender, depth, stage, or histology (Table II).

Table II shows the results of univariate and multivariate analyses of factors related to patients' prognosis. Univariate analysis showed that the phospho-PRAS40 Thr246-positive group had significantly poorer survival than the negative group $(p<0.001)$ (Figure 3). Furthermore, multivariate analysis indicated that phospho-PRAS40 Thr246 expression was one of the independent prognostic factors of overall survival for the patients with gastric cancer $(p=0.004)$.

\section{Discussion}

This present study immunohistochemically investigated the expression of phospho-PRAS40 Thr246 in gastric cancer. We found that more than half of the primary gastric cancers demonstrated positive phospho-PRAS40 Thr246 expression, and its expression correlated with lymph node metastasis and venous invasion, but not with other clinicopathological factors. Furthermore, phospho-PRAS40 Thr246 expression predicted poor prognosis, and is a novel prognostic factor for patients with gastric cancers.

Proline-rich Akt substrate (PRAS40) is a $40 \mathrm{kDa}$ substrate of Akt, and is also a negative regulator of mTOR and Akt activity. Activated Akt phosphorylates PRAS40 on threonine 246 , which in turn permits mTOR phosphorylation and activation [10, 11]. Nascimento et al. [12] indicated that phosphorylation of PRAS40 on Thr246 by Akt facilitates efficient phosphorylation of Ser183 by mTORC1. Increased PRAS40 phosphorylation has been associated with rising Akt activity in multiple cancer cells. Recently, phospho-PRAS40 ${ }^{\text {Thr2 } 46}$ has been further identified to be a biomarker for general PI3K pathway activation and predicts AKT inhibitor sensitivity, which further broadens its utility in the classification of cancer patients [8]. Several clinical studies have investigated the expression of phosphoPRAS40 Thr246 in human cancers. For example, Cloughesy et al. [13] showed that induction of phosphoPRAS40 ${ }^{\text {Thr } 246}$ is significantly associated with shorter time to progression in PTEN-deficient glioblastoma patients. McBride et al. [14] identified that there was a trend towards decreased survival in lowgrade glioma patients expressing phosphoPRAS40 Thr246. These findings were consistent with our results in gastric cancer. For the first time, in this study, the clinicopathological significance of phospho-PRAS40 Thr246 as a biomarker for general PI3K pathway activation was reported. Our findings are consistent with prior research emphasizing the value of clinical data in perioperative risk stratification, and further support the idea that PI3K inhibitors should be effective for the treatment of gastric cancer.

Considering the role of phospho-PRAS40 ${ }^{\text {Thr2 }} 46$ as a biomarker for pathway activation and AKT inhibitors sensitivity, our results indicated that the PI3K pathway would be frequently activated in gastric cancer, and a significant proportion (about 50\%) of patients with poor prognosis might benefit from Akt inhibitors. We suggest that therapeutic strategies targeting the PI3K/Akt pathway could be considered in the future treatment of gastric cancer. Furthermore, p-PRAS40 ${ }^{\text {Thr246 }}$ could also be used as a novel biomarker for the prognosis and classification of patients with gastric cancer.

\section{Acknowledgments}

The work was supported by The Xiamen Science and Technology Project (3502Z20104030). Yi-Zhuo Lu and An-mei Deng - equal contributors. 


\section{References}

1. Crew KD, Neugut Al. Epidemiology of gastric cancer. World J Gastroenterol 2006; 12: 354-62.

2. Li J, Yen C, Liaw D, et al. PTEN, a putative protein tyrosine phosphatase gene mutated in human brain, breast, and prostate cancer. Science 1997; 275: 1943-7.

3. Samuels $Y$, Wang Z, Bardelli A, et al. High frequency of mutations of the PIK3CA gene in human cancers. Science 2004; 304: 554.

4. Wojciechowska-Durczyńska K, Lewiński A. Phosphoinositide 3-kinase alterations in the thyroid gland a review study. Arch Med Sci 2009; 5: 10-5.

5. Courtney KD, Corcoran RB, Engelman JA. The PI3K pathway as drug target in human cancer. J Clin Oncol 2010; 28: 1075-83.

6. Arkenau HT. Gastric cancer in the era of molecularly targeted agents: current drug development strategies. J Cancer Res Clin Oncol 2009; 135: 855-66.

7. Tan DS, Thomas GV, Garrett MD, et al. Biomarker-driven early clinical trials in oncology: a paradigm shift in drug development. Cancer J 2009; 15: 406-20.

8. Andersen JN, Sathyanarayanan S, Di Bacco A, et al. Pathway-based identification of biomarkers for targeted therapeutics: personalized oncology with PI3K pathway inhibitors. Sci Transl Med 2010; 2: 43-55.

9. Cloughesy TF, Yoshimoto K, Nghiemphu P, et al. Antitumor activity of rapamycin in a Phase I trial for patients with recurrent PTEN-deficient glioblastoma. PLoS Med 2008; 5: e8.

10. Mamane $\mathrm{Y}$, Petroulakis E, LeBacquer O, Sonenberg N. mTOR, translation initiation and cancer. Oncogene 2006; 25: 6416-22.

11. Sabatini DM. mTOR and cancer: insights into a complex relationship. Nat Rev Cancer 2006; 6: 729-34.

12. Nascimento EB, Snel M, Guigas B, et al. Phosphorylation of PRAS40 on Thr246 by PKB/AKT facilitates efficient phosphorylation of Ser183 by mTORC1. Cell Signal 2010; 22: 961-7.

13. Cloughesy TF, Yoshimoto K, Nghiemphu P, et al. Antitumor activity of rapamycin in a Phase I trial for patients with recurrent PTEN-deficient glioblastoma. PLoS Med 2008; 5: e8.

14. McBride SM, Perez DA, Polley MY, et al. Activation of PI3K/ mTOR pathway occurs in most adult low-grade gliomas and predicts patient survival. J Neurooncol 2010; 97: 33-40. 